\title{
Stopping versus continuing long-term mepolizumab treatment in severe eosinophilic asthma (COMET study)
}

\author{
Wendy C. Moore ${ }^{1}$, Oliver Kornmann ${ }^{2}$, Marc Humbert $\mathbb{1}^{3,4,5}$, Claude Poirier $^{6}$, Elisabeth H. Bel ${ }^{7}$, \\ Norihiro Kaneko $\mathbb{( i )}^{8}$, Steven G. Smith ${ }^{9}$, Neil Martin ${ }^{10,11}$, Martyn J. Gilson ${ }^{12}$, Robert G. Price $\mathbb{1}^{13}$, \\ Eric S. Bradford ${ }^{9}$ and Mark C. Liu ${ }^{14}$
}

\begin{abstract}
${ }^{1}$ Dept of Medicine, Wake Forest School of Medicine, Medical Center Boulevard, Winston-Salem, NC, USA. ${ }^{2}$ IKF Pneumologie Frankfurt, Clinical Research Centre Respiratory Diseases, Frankfurt, Germany. ${ }^{3}$ Université Paris-Saclay, Paris, France. ${ }^{4}$ Assistance PubliqueHôpitaux de Paris, Service de Pneumologie et Soins Intensifs Respiratoires, Hôpital Bicêtre, Le Kremlin-Bicêtre, France. ${ }^{5}$ INSERM U999, Le Kremlin-Bicêtre, Paris, France. ${ }^{6}$ Département de Médecine, Service de Pneumologie, Centre Hospitalier de l'Université de Montréal (CHUM), Montreal, QC, Canada. ${ }^{7}$ Dept of Respiratory Medicine, Amsterdam UMC, University of Amsterdam, Amsterdam, The Netherlands. ${ }^{8}$ Dept of Pulmonary Medicine, Kameda Medical Center, Kamogawa, Japan. ${ }^{9}$ Respiratory Therapeutic Area, GSK, Research Triangle Park, NC, USA. ${ }^{10}$ Global Medical Affairs, GSK, Brentford, UK. ${ }^{11}$ Institute for Lung Health, University of Leicester, Leicester, UK. ${ }^{12}$ Respiratory Research and Development, GSK, Uxbridge, UK. ${ }^{13}$ Biostatistics, GSK, Stevenage, UK. ${ }^{14}$ Divisions of Allergy and Clinical Immunology, Pulmonary and Critical Care Medicine, Johns Hopkins Asthma and Allergy Center, Baltimore, MD, USA.
\end{abstract}

Corresponding author: Wendy C. Moore (wmoore@wakehealth.edu)

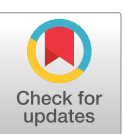

Copyright @The authors 2022

This version is distributed under the terms of the Creative Commons Attribution Non-Commercial Licence 4.0. For commercial reproduction rights and permissions contact permissions@ersnet.org

This article has supplementary material available from erj.ersjournals.com

Received: 8 Feb 2021 Accepted: 23 May 2021

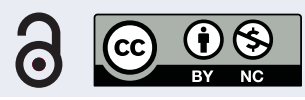

Shareable abstract (@ERSpublications)

This randomised study demonstrates increased exacerbation risk and a decrease in asthma control in patients with severe eosinophilic asthma who stop mepolizumab treatment after long-term use, when compared with those who continue treatment. https://bit.ly/3fsxGV2

Cite this article as: Moore WC, Kornmann O, Humbert M, et al. Stopping versus continuing long-term mepolizumab treatment in severe eosinophilic asthma (COMET study). Eur Respir J 2022; 59: 2100396 [DOI: 10.1183/13993003.00396-2021].

\section{Abstract}

Background The long-term efficacy and safety of mepolizumab for treatment of severe eosinophilic asthma are well established. Here, we examine the clinical impact of stopping mepolizumab after long-term use.

Methods COMET (NCT02555371) was a randomised, double-blind, placebo-controlled, parallel-group, multicentre study. Patients who had completed COLUMBA (NCT01691859) or COSMEX (NCT02135692) and received continuous mepolizumab treatment for $\geqslant 3$ years were randomised 1:1 to stop (switch to placebo) or continue subcutaneous mepolizumab $100 \mathrm{mg}$ every 4 weeks for 52 weeks. Primary end-point: time to first clinically significant exacerbation; secondary end-points: time to first exacerbation requiring hospitalisation/emergency department visit, time to decrease in asthma control ( $\geqslant 0.5$-point increase in Asthma Control Questionnaire-5 score from COMET baseline) and blood eosinophil count ratio to COMET baseline. Safety was assessed.

Results Patients stopping ( $\mathrm{n}=151)$ versus continuing $(\mathrm{n}=144)$ mepolizumab had significantly shorter times to first clinically significant exacerbation (hazard ratio 1.61, 95\% CI 1.17-2.22; $\mathrm{p}=0.004$ ) and decrease in asthma control (hazard ratio 1.52, 95\% CI 1.13-2.02; p=0.005), and higher blood eosinophil counts at week 52 (270 versus 40 cells $\mu \mathrm{L}^{-1}$; ratio (stopping versus continuing) 6.19, 95\% CI 4.89-7.83; $\mathrm{p}<0.001$ ). Differences in efficacy outcomes between groups were observed when assessed from week 12 (16 weeks after last mepolizumab dose). Exacerbations requiring hospitalisation/emergency department visit were rare. Adverse events in patients continuing mepolizumab were consistent with previous studies. For patients who stopped mepolizumab, the safety profile was consistent with other eosinophilic asthma populations.

Conclusion Patients who stopped mepolizumab had an increase in exacerbations and reduced asthma control versus those who continued.

\section{Introduction}

Efficacy and safety of long-term treatment with the targeted, humanised, anti-interleukin (IL)-5 monoclonal antibody mepolizumab in patients with severe eosinophilic asthma have been demonstrated for up to 4.5 years in previous double-blinded [1-5] and open-label studies [6-8]. Mepolizumab is approved for the 
treatment of severe eosinophilic asthma in multiple regions worldwide, and for the treatment of eosinophilic granulomatosis with polyangiitis in adults in the USA and Japan and for the treatment of hypereosinophilic syndrome in patients aged $\geqslant 12$ years in the USA [9, 10]. Mepolizumab selectively binds to IL-5, inhibiting eosinophilic inflammation [11, 12]; however, it is currently unknown whether the suppression of eosinophilic airway inflammation seen with mepolizumab in severe eosinophilic asthma continues if patients stop treatment, or whether long-term treatment should be recommended for sustained disease control.

A previous 12-month observational follow-up study of patients who stopped mepolizumab $750 \mathrm{mg}$ intravenous treatment after 1 year suggested an increase in blood eosinophil counts to pre-treatment levels and a subsequent return to an exacerbating phenotype [13]. Understanding responses after stopping is of interest, as long-term treatment may have a sustained disease-modifying effect and potentially permanently reduce eosinophil counts [14]. However, there are no data on the impact of stopping in patients treated with mepolizumab for $>1$ year, which is important, as short-term discontinuation may be required for some patients in clinical practice. Additionally, there are no data on the impact of stopping the licensed mepolizumab dose in adults and adolescents, $100 \mathrm{mg}$ subcutaneously.

The aim of this randomised, placebo-controlled study was to evaluate the clinical impact of stopping mepolizumab $100 \mathrm{mg}$ s.c. in patients with severe eosinophilic asthma following long-term ( $\geqslant 3$ years) exposure by assessing exacerbation rates and other clinical parameters in those who stopped mepolizumab (and switched to placebo) versus those who continued. A visual summary of the COMET study is presented in supplementary figure E1.

\section{Materials and methods}

\section{Study design}

COMET (GSK ID 201810; NCT02555371) was a global, multicentre, randomised, double-blind, placebo-controlled, parallel-group study to compare stopping versus continuation of long-term mepolizumab treatment in patients with severe eosinophilic asthma.

The study comprised four parts (figure 1). Following screening, patients with $<3$ years of mepolizumab treatment entered part A, a variable open-label run-in period of $0-132$ weeks designed to ensure all patients had received continuous mepolizumab $100 \mathrm{mg}$ s.c. (in addition to standard of care) for $\geqslant 3$ years. Once patients had $\geqslant 3$ years of mepolizumab exposure they entered part $B$, which consisted of a fixed open-label run-in of 4-8 weeks during which open-label mepolizumab (100 mg s.c. every 4 weeks plus standard of care) was administered and baseline information was collected. Patients who had $\geqslant 3$ years of mepolizumab treatment at screening entered part B directly. Following part B, patients were randomised 1:1 to stop mepolizumab treatment and switch to placebo (plus standard of care) or receive continued mepolizumab $100 \mathrm{mg}$ s.c. plus standard of care every 4 weeks for 52 weeks (part C). Patients with one or more clinically significant exacerbation (supplementary material) during part $\mathrm{C}$ were assessed by the study investigator to determine whether the patient should continue taking double-blind study treatment (continue in part C) or return to open-label mepolizumab $100 \mathrm{mg}$ s.c. every 4 weeks (switch to part D; optional, see supplementary material) for the remainder of the study (up to 52 weeks post-randomisation). No formal criteria for treatment continuation were provided to investigators; the decision was based on the opinion of the treating physician in collaboration with the patient. Patients remained on a stable standard of care asthma therapy from the start of part B to the end of the study. The reported results focus mainly on the blinded, randomised part C.

The study was conducted in accordance with International Conference on Harmonisation good clinical practice, all applicable patient privacy requirements, and the guiding principles of the current version of the Declaration of Helsinki. All patients provided written informed consent prior to study participation.

\section{Patients}

Patients with severe eosinophilic asthma who had completed one of the previous open-label studies, COLUMBA (MEA115666; NCT01691859) [7] or COSMEX (201312; NCT02135692) [8], were eligible for COMET (supplementary materials). To enter the study, patients had to have completed and received continuous mepolizumab treatment (i.e. no treatment gaps $>12$ weeks (84 days, equivalent to at least two consecutive missed doses) between any two mepolizumab doses), and remained adherent on asthma controller medication/therapy, throughout the previous open-label mepolizumab studies.

Exclusion criteria, randomisation criteria for entry to part $\mathrm{C}$ and details of randomisation and blinding are listed in the supplementary materials. 


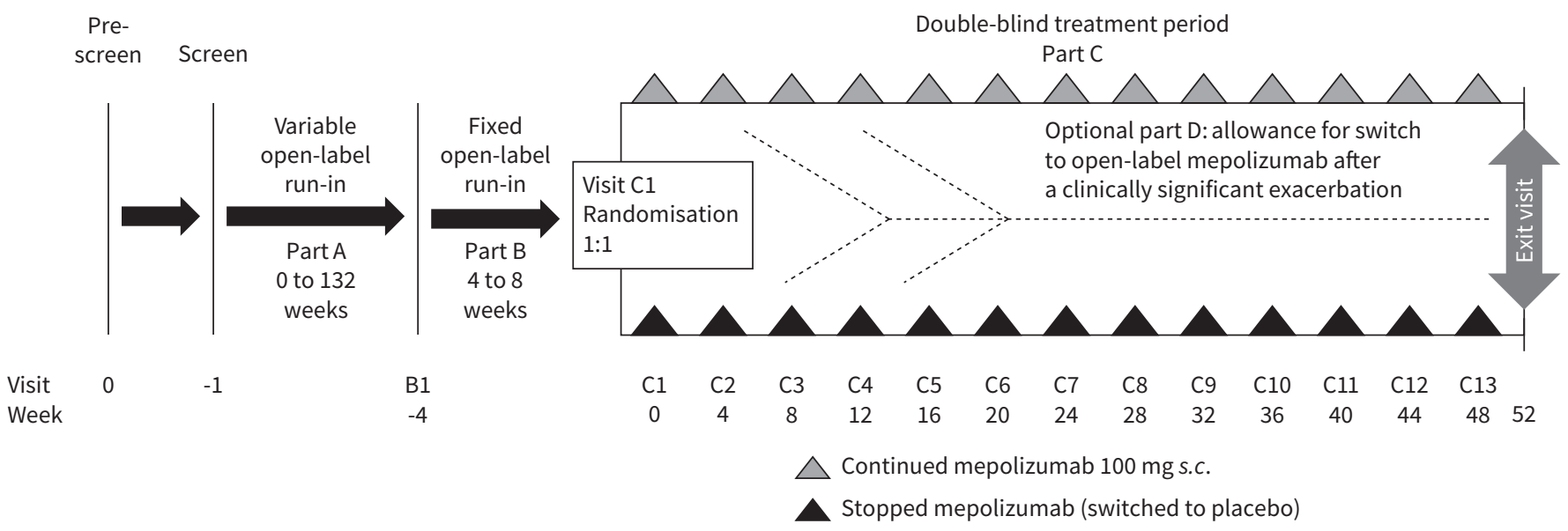

\begin{tabular}{|llll|}
\hline Phase & Phase title & Duration & Treatment arms \\
\hline Part A & Variable open-label run-in & $0-132$ weeks & $\begin{array}{l}\text { Open-label mepolizumab (100 mg s.c.) every 4 weeks } \\
\text { for patients with <3 years mepolizumab treatment }\end{array}$ \\
\hline Part B & Fixed open-label run-in & 4-8 weeks & Open-label mepolizumab (100 mg s.c.) every 4 weeks \\
\hline Part C & Double-blind treatment period & 52 weeks & $\begin{array}{l}\text { Patients randomised 1:1 to double-blind study treatment: } \\
\text { - Mepolizumab 100 mg s.c. every 4 weeks } \\
\text { Placebo administered s.c. every } 4 \text { weeks }\end{array}$ \\
\hline Part D & $\begin{array}{l}\text { Open-label switch } \\
\text { (optional following exacerbation) }\end{array}$ & $\begin{array}{l}\text { Up to } 52 \text { weeks } \\
\text { post-randomisation }\end{array}$ & Open-label mepolizumab (100 mg s.c.) every 4 weeks \\
\hline
\end{tabular}

FIGURE 1 Study design and description of the individual study parts.

\section{End-points and assessments}

The primary end-point was time to the first clinically significant exacerbation during part C (post-randomisation). Secondary end-points were time to the first exacerbation requiring hospitalisation or emergency department (ED) visit; time to a decrease in asthma control, defined as an increase from baseline (start of double-blind treatment in COMET) in Asthma Control Questionnaire (ACQ)-5 score of $\geqslant 0.5$ units [15]; and ratio to baseline in blood eosinophil count at weeks 12, 24, 36 and 52. Other end-points included time to the first exacerbation requiring hospitalisation, mean change from baseline in ACQ-5 score assessed every 4 weeks until week 52 and mean change from baseline in health-related quality of life (as assessed by St George's Respiratory Questionnaire (SGRQ) total score) and clinic pre-bronchodilator forced expiratory volume in $1 \mathrm{~s}\left(\mathrm{FEV}_{1}\right)$ at weeks 12, 24, 36 and 52. Safety end-points included adverse events and serious adverse events, clinical laboratory parameters, 12-lead ECG parameters and vital signs.

\section{Sample size and statistical analysis}

The sample size was based on the primary end-point of time to first clinically significant exacerbation to test the superiority of continued mepolizumab treatment compared with stopping mepolizumab (randomised to placebo).

The intent-to-treat population, used for all efficacy and safety data analyses, comprised all randomised patients who received one or more dose of double-blind study treatment. For all change from/ratio to baseline end-points, baseline was defined as the start of double-blind treatment (randomisation) in COMET. Only relevant data from part $\mathrm{C}$ were included in the analysis of the double-blind period, with on-treatment data censored following the discontinuation of double-blind treatment or switch to open-label mepolizumab within part D. Further details of the sample size and statistical analyses are provided in the supplementary material. Additionally, a post hoc analysis was performed to evaluate whether baseline characteristics can identify patients with a greater or reduced treatment effect following stopping or 
continuing long-term mepolizumab treatment and further information can be found in the supplementary material.

Results

Patient population

Patient disposition from previous studies is shown in supplementary figure E2. Between January 2016 and November 2017, 306 patients were enrolled in parts A/B and 295 patients were randomised in part C (placebo $n=151$; mepolizumab $n=144$ ) (supplementary figure E3). Of these, 129 patients, 84 who had received placebo in part $\mathrm{C}$ and 45 who had received mepolizumab in part $\mathrm{C}$ switched to open-label mepolizumab (part D) following an asthma exacerbation (supplementary figure E4); the most common reason for switching in both groups was a reported lack of efficacy in the opinion of the investigator ( $\mathrm{n}=81$ and $n=42$, where $n=81$ and $n=41$ reported exacerbation as a subreason, respectively). The study was completed in July 2019.

Demographic and disease characteristics at the first part $\mathrm{C}$ visit were similar between the placebo and mepolizumab groups (table 1). The geometric mean blood eosinophil counts were similar in both groups at the start of part $\mathrm{C}$ (placebo 40 cells $\cdot \mu \mathrm{L}^{-1}$; mepolizumab 50 cells $\cdot \mu \mathrm{L}^{-1}$ ), and were decreased compared with baseline values before any mepolizumab treatment in DREAM, MENSA and SIRIUS (230-320 cells $\mu \mathrm{L}^{-1}$; supplementary table E2). In addition, demographic and disease characteristics were similar before any treatment with mepolizumab for each group in COMET (e.g. at DREAM, MENSA and SIRIUS baseline; supplementary table E3).

TABLE 1 Demographics and asthma characteristics of patients on long-term mepolizumab at first clinic visit in part C and study treatment exposure

\begin{tabular}{|c|c|c|c|}
\hline & $\begin{array}{l}\text { Stopped mepolizumab } \\
\text { (switched to placebo) }\end{array}$ & $\begin{array}{l}\text { Continued mepolizumab } \\
100 \mathrm{mg} \text { s.c. }\end{array}$ & Total \\
\hline Patients, $n$ & 151 & 144 & 295 \\
\hline Females, n (\%) & $86(57)$ & $87(60)$ & $173(59)$ \\
\hline Age, years, mean \pm sD & $55.7 \pm 11.42$ & $56.6 \pm 11.53$ & $56.1 \pm 11.46$ \\
\hline \multicolumn{4}{|l|}{ Race, $n(\%)$} \\
\hline Asian & $24(16)$ & $24(17)$ & $48(16)$ \\
\hline Black & $2(1)$ & $5(3)$ & $7(2)$ \\
\hline White & $125(83)$ & $115(80)$ & $240(81)$ \\
\hline Body mass index, $\mathrm{kg} \cdot \mathrm{m}^{-2}$, mean $\pm \mathrm{sD}$ & $28.0 \pm 5.63$ & $29.1 \pm 6.29$ & $28.5 \pm 5.98$ \\
\hline Duration of asthma, years, mean \pm sD & $22.8 \pm 13.82$ & $25.1 \pm 14.54$ & $23.9 \pm 14.20$ \\
\hline \multicolumn{4}{|l|}{ Current concomitant medication", n (\%) } \\
\hline LABA & $145(96)$ & $141(98)$ & $286(97)$ \\
\hline ICS & $146(97)$ & $137(95)$ & $283(96)$ \\
\hline Using maintenance OCS, $\mathrm{n}(\%)$ & $17(11)$ & $21(15)$ & $38(13)$ \\
\hline Dose, $\mathrm{mg} \cdot \mathrm{day}^{-1}$ (prednisone equivalent), median (range) & $5.0(0.0-20.0)$ & $5.0(0.0-20.0)$ & $5.0(0.0-20.0)$ \\
\hline Blood eosinophil count, cells $\mu \mathrm{L}^{-1}$, geometric mean \pm SD of log & $40 \pm 0.870$ & $50 \pm 0.881$ & $50 \pm 0.876$ \\
\hline Exacerbations in previous year, mean $\pm \mathrm{sD}$ & $0.6 \pm 1.08$ & $0.8 \pm 1.51$ & $0.7 \pm 1.31$ \\
\hline Exacerbations requiring hospitalisation or ED visit in the previous year, $n(\%)$ & $5(3)$ & $5(3)$ & $10(3)$ \\
\hline Exacerbations requiring hospitalisation in the previous year, $\mathrm{n}(\%)$ & $4(3)$ & $3(2)$ & $7(2)$ \\
\hline ACQ-5 score, mean \pm SD & $1.2 \pm 1.04$ & $1.4 \pm 1.05$ & $1.3 \pm 1.05$ \\
\hline SGRQ total score, mean \pm SD & $32.2 \pm 17.82$ & $33.1 \pm 17.42$ & $32.7 \pm 17.60$ \\
\hline Pre-bronchodilator $\mathrm{FEV}_{1}, \mathrm{~mL}$, mean $\pm \mathrm{SD}$ & $1921 \pm 655$ & $1774 \pm 666$ & $1849 \pm 663$ \\
\hline Pre-bronchodilator $\mathrm{FEV}_{1}, \%$ predicted, mean $\pm \mathrm{SD}$ & $65.5 \pm 19.64$ & $61.6 \pm 19.08$ & $63.6 \pm 19.43$ \\
\hline \multicolumn{4}{|l|}{ Mepolizumab continuous exposure prior to randomisation at visit $\mathrm{C} 1$} \\
\hline Time on mepolizumab, months, median (range) & $44.1(36-59)$ & $43.6(32-58)$ & $44.1(32-59)$ \\
\hline Total exposure, patient-years & 588.0 & 557.2 & 1145.2 \\
\hline \multicolumn{4}{|l|}{ Smoking history, $\mathrm{n}(\%)$} \\
\hline Never-smoker & $128(85)$ & $123(85)$ & $251(85)$ \\
\hline Former smoker & $23(15)$ & $21(15)$ & $44(15)$ \\
\hline \multicolumn{4}{|l|}{ Study treatment exposure during COMET (part C) } \\
\hline Total exposure, patient-years & 93.9 & 114.6 & 208.5 \\
\hline
\end{tabular}


Prior to randomisation in part C, the median (range) duration of mepolizumab treatment was 44.1 (32-59) months, equating 1145.2 patient-years of exposure (table 1). Two patients who had 32 months of continuous mepolizumab exposure were randomised prematurely to part $\mathrm{C}$ and subsequently identified as protocol deviations. During the double-blind part $\mathrm{C}$ period, a greater proportion of patients in the placebo arm (56\%, 84 out of 151) switched to open-label mepolizumab treatment (part D) than in the mepolizumab arm (31\%, 45 out of 144; supplementary figures E3 and E4). Consequently, the total exposure to double-blind treatment was longer for the mepolizumab group (114.6 patient-years) compared with placebo (93.9 patient-years; table 1).

\section{Primary end-point}

Patients who stopped mepolizumab had a significantly shorter time to first clinically significant exacerbation than patients who continued; they had a $61 \%$ increase in the risk of experiencing their first clinically significant exacerbation (stopping/continuing mepolizumab hazard ratio (HR) 1.61, 95\% CI 1.17-2.22; $\mathrm{p}=0.004$ ) over the 52-week part C period (figure 2a). A significantly higher proportion of patients who stopped mepolizumab (placebo group) experienced at least one clinically significant exacerbation during part $\mathrm{C}$ compared with those continuing mepolizumab treatment (59\% versus 46\%; OR 1.99, 95\% CI 1.19-3.32; p=0.009; supplementary table E4). The Kaplan-Meier cumulative incidence of patients experiencing an exacerbation was numerically higher with placebo versus mepolizumab, respectively, at each time point: between-treatment differences were detected from week 12 (31.8\% and 20.2\%), and grew progressively greater through week 24 (49.3\% and 32.3\%), week 36 (56.0\% and 40.3\%) and week 52 (60.7\% and 47.1\%). Overall, 22 (15\%) patients in the placebo group and 19 (13\%) patients in the mepolizumab group experienced two or more exacerbations while on treatment during part C.

\section{Secondary and other end-points}

Exacerbations requiring hospitalisation or ED visit occurred in seven (5\%) patients who stopped mepolizumab treatment (nine events; one patient experienced three events requiring ED visit and was never switched to part D; six patients experienced a single exacerbation requiring hospitalisation, five of whom were immediately switched to part D). For patients who continued mepolizumab, 10 (7\%) experienced a single event of this severity (eight patients had an ED visit, seven of whom were switched to part D; the remaining two patients required hospitalisation and both were immediately switched to part D).

Patients who stopped mepolizumab treatment had a significantly shorter time to first decrease in asthma control during part C; they had a 52\% increase in risk of having a decrease in asthma control over the 12-month period (stopping/continuing mepolizumab HR 1.52, 95\% CI 1.13-2.02; $\mathrm{p}=0.005$; figure 2b). The Kaplan-Meier cumulative incidence of patients experiencing a decrease in asthma control was higher with

a)

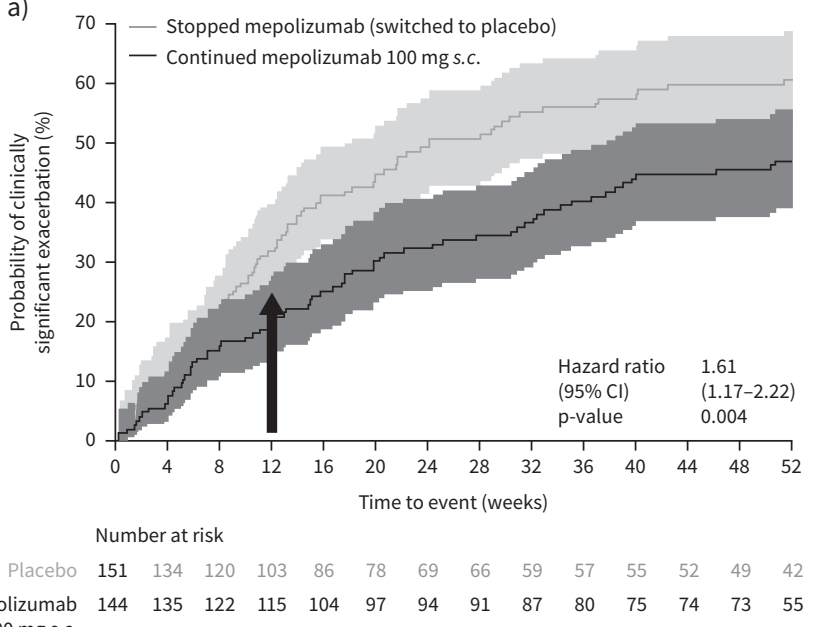

b)

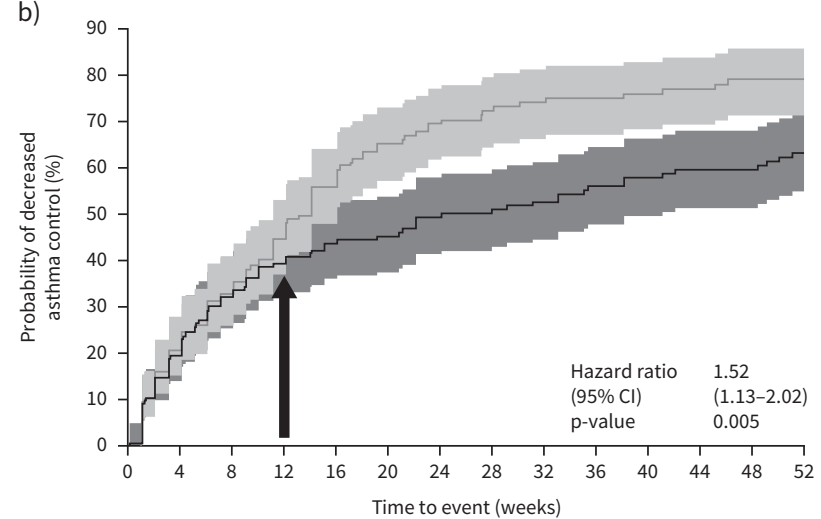

Number at risk

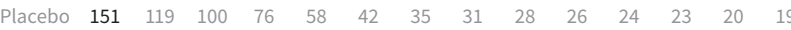
$\begin{array}{lllllllllllllll}\text { Mepolizumab } & 144 & 116 & 96 & 84 & 73 & 70 & 62 & 60 & 57 & 50 & 48 & 46 & 45 & 32\end{array}$ $100 \mathrm{mg}$ S.C.

FIGURE 2 Kaplan-Meier cumulative incidence curve for time to first a) clinically significant exacerbation; and b) decrease in asthma control, defined as an increase from baseline in Asthma Control Questionnaire- 5 score of $\geqslant 0.5$ units [15], and the associated hazard ratios (stopping/ continuing mepolizumab) (on-treatment; Part C; blinded treatment). Week 0 represents 4 weeks following the last dose of open-label mepolizumab. Shaded areas represent 95\% confidence intervals. Arrows indicate that the difference between groups was seen from week 12 onwards (16 weeks after the last dose of open-label mepolizumab). 
placebo versus mepolizumab, respectively, at each time point: between-treatment differences were detected from week 12 (44.5\% and 39.3\%) and increased through week 24 (69.5\% and 49.3\%), week 36 (74.9\% and 56.0\%) and week 52 (79.0\% and 63.1\%). Worsening asthma control, as indicated by increased ACQ-5 scores, was seen from week 12 (16 weeks after the last dose of open-label mepolizumab) in patients who stopped mepolizumab treatment compared with those who continued, although 95\% confidence intervals overlapped at each time point (figure 3a). By week 52, the difference (95\% CI) in ACQ-5 scores for patients stopping versus continuing mepolizumab was $0.23(-0.02-0.48 ; \mathrm{p}=0.067)$.

In patients who stopped mepolizumab, least squares (LS) mean \pm SE logs blood eosinophil counts increased steadily from week 4 (8 weeks after the last dose of open-label mepolizumab), reaching $270 \pm 0.077$ cells· $\mu \mathrm{L}^{-1}$ by week 12 (16 weeks after patients' last dose of open-label mepolizumab), while patients continuing mepolizumab maintained their LS mean blood eosinophil count at $40-60$ cells $\cdot \mu \mathrm{L}^{-1}$ (week 12 stopping versus

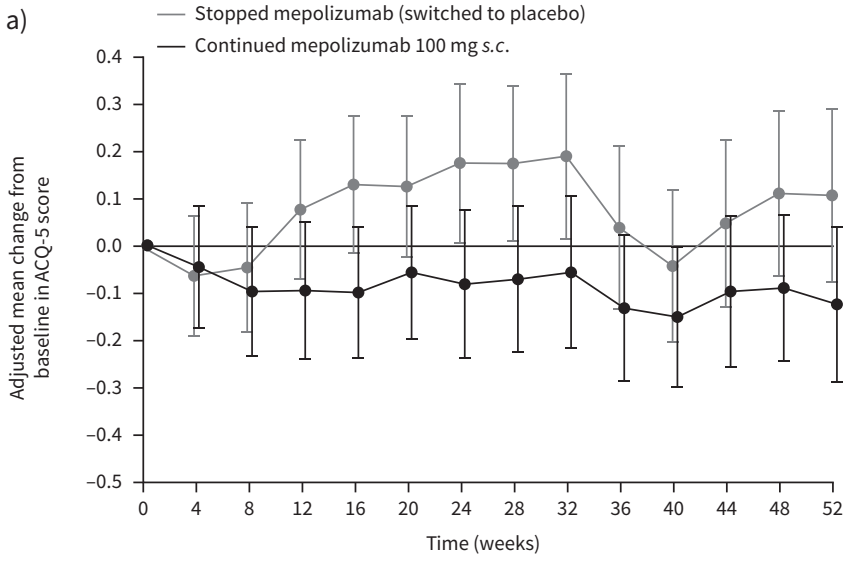

Number of patients at each time point

$\begin{array}{lllllllllllllll}\text { Placebo } & 151 & 145 & 132 & 125 & 106 & 91 & 84 & 81 & 76 & 69 & 68 & 66 & 61 & 56\end{array}$ $\begin{array}{lllllllllllllll}\text { Mepolizumab } & 144 & 143 & 134 & 130 & 124 & 117 & 110 & 107 & 105 & 98 & 95 & 96 & 93 & 74\end{array}$ $100 \mathrm{mg}$ s.c.

c)

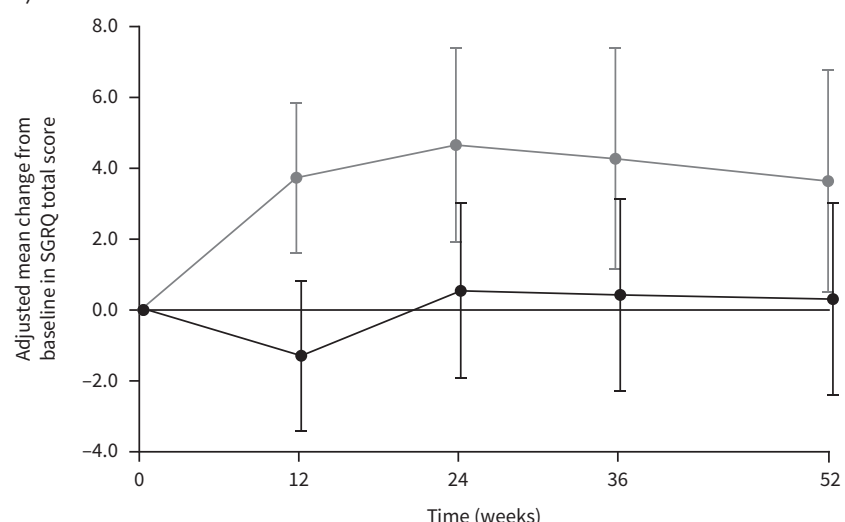

Number of patients at each time point

$\begin{array}{rrrrrr}\text { Placebo } & 151 & 137 & 92 & 74 & 65 \\ \text { Mepolizumab } & 144 & 141 & 117 & 106 & 94 \\ 100 \mathrm{mg} \text { S.c. } & & & & & \end{array}$

b)

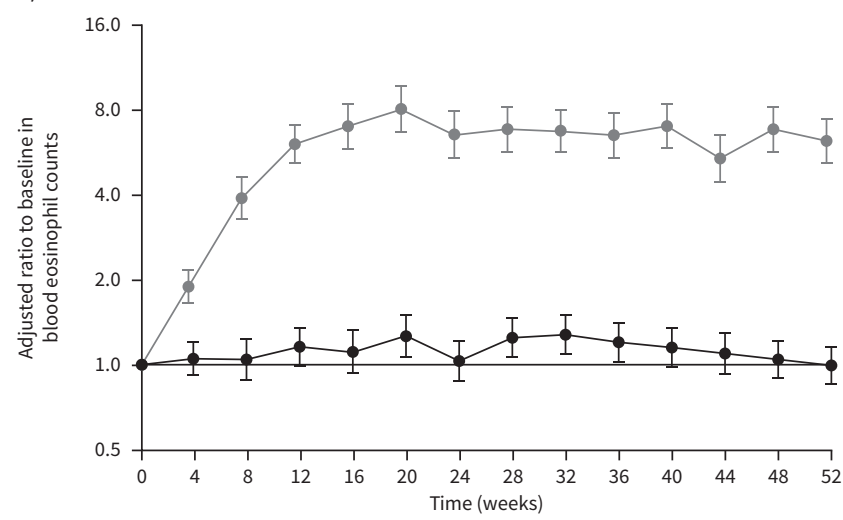

Number of patients at each time point

$\begin{array}{lllllllllllllll}\text { Placebo } & 150 & 145 & 136 & 121 & 103 & 84 & 79 & 77 & 75 & 65 & 68 & 66 & 60 & 60\end{array}$ $\begin{array}{lllllllllllllll}\text { Mepolizumab } & 141 & 137 & 133 & 120 & 114 & 102 & 106 & 101 & 99 & 99 & 93 & 92 & 86 & 92\end{array}$ $100 \mathrm{mg}$ s.c.

\section{d)}

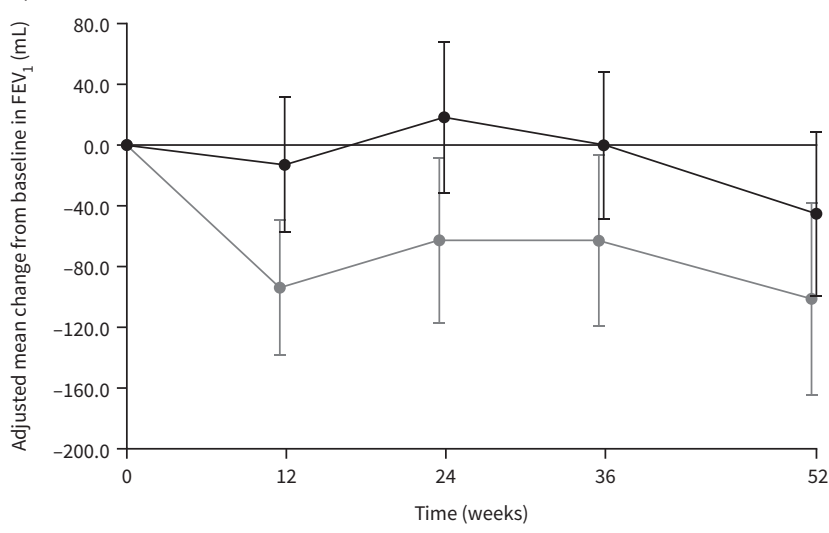

Number of patients at each time point

\begin{tabular}{|c|c|c|c|c|}
\hline Placebo & 150 & 137 & 93 & 75 \\
\hline $\begin{array}{l}\text { epolizumab } \\
100 \mathrm{mg} \text { s.c. }\end{array}$ & 144 & 139 & 116 & 106 \\
\hline
\end{tabular}

FIGURE 3 a) Mean change from baseline (start of double-blind treatment in COMET) in Asthma Control Questionnaire (ACQ)-5 score; b) ratio to baseline in blood eosinophil count; c) mean change from baseline in St George's Respiratory Questionnaire (SGRQ) total score; and d) mean

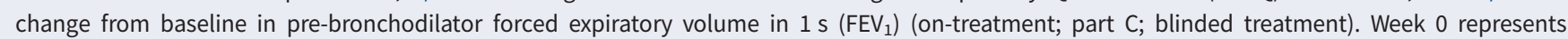
4 weeks following the last dose of open-label mepolizumab. Data are presented as least squares means $(95 \% \mathrm{Cl})$. Patient numbers at each given time point are shown below each graph. Higher scores on the ACQ-5 indicate worse control (range 0-6); a change of 0.5 points is the minimal clinically important difference [15]. Higher scores on the SGRQ indicate a worse function (range 0-100); a change of 4 points is the minimal clinically important difference [16]. 
continuing mepolizumab ratio 5.21, 95\% CI 4.20-6.46; $\mathrm{p}<0.001$ ). By week 52, blood eosinophil counts were $270 \pm 0.091$ cells $\cdot \mu \mathrm{L}^{-1}$ and $40 \pm 0.077$ cells $\cdot \mu \mathrm{L}^{-1}$ for patients stopping versus continuing mepolizumab, respectively (stopping versus continuing mepolizumab ratio 6.19, 95\% CI 4.89-7.83; p<0.001) (figure 3b). Absolute blood eosinophil counts are shown in supplementary table E5.

Health-related quality of life (change from baseline in SGRQ total score) and lung function (change from baseline in pre-bronchodilator $\mathrm{FEV}_{1}$ ) were measured at weeks 12, 24, 36 and 52, with worsening seen for both end-points at all time points in patients who stopped mepolizumab; patients who continued mepolizumab experienced sustained benefit in these outcomes, although 95\% confidence intervals overlapped at most time points (figure 3c,d). By week 52, the difference (95\% CI) in SGRQ total scores and pre-bronchodilator $\mathrm{FEV}_{1}$ for patients stopping versus continuing mepolizumab was 3.3 (-0.8-7.5; $\mathrm{p}=0.113)$ and $-56 \mathrm{~mL}(-139-27 \mathrm{~mL} ; \mathrm{p}=0.186)$.

\section{Post hoc analysis}

Our post hoc analysis showed that the number of exacerbations in the year prior to randomisation $(0,1$, $\geqslant 2$ ) was a strong prognostic factor for risk of exacerbation during the 52-week trial period, with an increasing risk of exacerbation during part $\mathrm{C}$ associated with a greater number of exacerbation events in the year prior to randomisation. Patients with two or more exacerbations in the previous year $(n=44)$ had a higher risk of exacerbation versus those with no $(n=185)$ or one $(n=66)$ exacerbation (supplementary figure E5). Additionally, stopping versus continuing mepolizumab increased the risk of first exacerbation by $74 \%$ (HR 1.74, 95\% CI 1.08-2.80) and 180\% (2.80, 1.44-5.44), respectively, in patients who had no or one exacerbation in the previous year; however, this increased risk was not seen for patients with two or more exacerbations in the previous year $(0.88,0.46-1.67)$. No other baseline characteristic assessed identified a differential treatment effect across subgroups.

\section{Safety}

The safety profile of mepolizumab was consistent with previous trials (supplementary table E6). In part C, a lower proportion of patients on placebo reported adverse events than those on mepolizumab (64\% versus 78\%); however, exposure per patient-year was lower in the placebo group than in the mepolizumab group (93.94 versus 114.60). Exposure-adjusted rates (per 1000 participant-years) were similar between the treatment arms (placebo 3097.77, mepolizumab 2740.01; supplementary table E7). A similar proportion of patients in the placebo and mepolizumab groups reported serious adverse events (7\% versus $6 \%$ ), none of which were considered by the treating investigator to be related to treatment. Infections were reported in a lower proportion of patients in the placebo group compared with the mepolizumab group (44\% versus 58\%), with similar exposure-adjusted rates between the treatment arms (1373.24 and 1160.58 per 1000 participant-years, respectively). Local site reactions occurred in a lower proportion of placebo-treated versus mepolizumab-treated patients $(<1 \%$ versus $3 \%)$. There were two reported deaths, one during part C (post-treatment, unknown cause) and one during part D (on-treatment, pneumonia aspiration); both deaths were in patients who received placebo during part $\mathrm{C}$ and both were considered to be unrelated to study treatment.

\section{Discussion}

This is the first randomised study to examine the clinical impact of stopping mepolizumab treatment after long-term use in patients with severe eosinophilic asthma. The time to first clinically significant exacerbation was significantly shorter for those who stopped mepolizumab compared with those who continued mepolizumab treatment. 59\% of patients who stopped mepolizumab experienced at least one clinically significant exacerbation in the following year, compared with $46 \%$ of those who remained on mepolizumab. Worsening of ACQ-5 and SGRQ scores were seen at week 12 (16 weeks after the last dose of open-label mepolizumab), whereas scores were maintained in those who continued mepolizumab. This suggests a loss of asthma control and quality of life in patients who stop mepolizumab treatment, even after $\geqslant 3$ years of use. Therefore, the beneficial effects of mepolizumab, evident in the baseline characteristics at the start of double-blind treatment in COMET, are unlikely to be sustained after stopping treatment. These results also highlight the sustained clinical benefit in patients who remain on mepolizumab treatment. Overall, compared with patients who stopped mepolizumab, patients who continued long-term treatment were less likely to experience exacerbations and loss of asthma control, with sustained benefits also shown in health-related quality of life and lung function. The probability of a clinically significant exacerbation and decreased asthma control appeared to increase from around week 12 (16 weeks after the last dose of open-label mepolizumab) for patients who stopped mepolizumab versus those who continued treatment. 
There were differences in blood eosinophil counts between those who stopped mepolizumab and those who continued, in accordance with the mechanism of action of mepolizumab [9]; these differences were seen as early as week 4 ( 8 weeks after the last dose of open-label mepolizumab). Blood eosinophil counts increased slowly; by week 12 (16 weeks after stopping mepolizumab) they had returned to values similar to baseline at the start of the initial phase III studies (270 cells $\mu \mathrm{L}^{-1}$ versus $230-320$ cells $\left.\mu \mathrm{L}^{-1}\right)[1,3,4]$. This is consistent with the publication by HALDAR et al. [13] which demonstrated a return to pre-treatment blood eosinophil counts after stopping therapy. Importantly, changes in blood eosinophil counts preceded the separation in efficacy between the placebo and mepolizumab arms during part C. This supports previous findings demonstrating that the pharmacodynamic effects of mepolizumab on blood eosinophil counts are not sustained after stopping treatment [13], and further confirms the association between clinical outcomes and blood eosinophil counts [1, 3, 4, 17-20].

Although 59\% of patients who stopped mepolizumab experienced at least one clinically significant exacerbation in the year after stopping treatment, $41 \%$ experienced no clinically significant exacerbations during this period. However, this is consistent with the proportion of patients who experienced no exacerbations while receiving placebo during the previous mepolizumab trials $[1,3-5]$, and in a similar 12-week trial of dupilumab [21]. For example, in MENSA, 45\% of patients receiving placebo did not experience an exacerbation over the 32-week treatment period [4], while the equivalent proportions from SIRIUS (24 weeks), DREAM (52 weeks), MUSCA (24 weeks) and the dupilumab trial (12 weeks) were $32 \%, 33 \%, 59 \%$ and $56 \%$, respectively [1, 3, 5, 21]. These findings highlight the frequency of exacerbations in patients with a severe eosinophilic asthma phenotype. The fact that some patients experienced exacerbations while receiving mepolizumab treatment supports heterogeneity within the phenotype, whereby factors other than eosinophils may contribute to exacerbations [22]. Nonetheless, the stability of exacerbating asthma phenotypes over a given time period is currently unknown [23-26], and it is feasible that patients who have a history of severe exacerbations while on standard of care treatment may remain exacerbation free for indeterminant periods and then return to an exacerbating phenotype [27].

In the XPORT study, $48 \%$ of patients who stopped omalizumab treatment remained exacerbation free in the following year, similar to the $41 \%$ of patients in COMET who did not exacerbate after stopping mepolizumab [28]. As highlighted previously, this is likely due to the variability of exacerbation incidence in the severe eosinophilic asthma population. Additionally, as shown in COMET, a higher proportion of patients who stayed on omalizumab (67\%) did not exacerbate in the 1-year study period, compared with those who stopped treatment (48\%) [28]. This is in line with the $54 \%$ of patients who remained exacerbation free while continuing on mepolizumab in COMET.

It may be useful to determine the characteristics or adherence of patients who did not return to an exacerbating phenotype within 12 months of stopping mepolizumab treatment, and indeed to determine whether these patients had any other markers of sustained treatment benefit outside of exacerbations. This information may then be used to determine whether there are any patients who can stop mepolizumab without any subsequent loss of control during a 52-week period after a sustained period of treatment, although clinical studies would be needed to confirm. It is therefore of interest that the number of exacerbations in the year prior to COMET randomisation was a strong prognostic factor to predict future exacerbation risk, and was the only baseline characteristic assessed that identified a differential treatment effect across subgroups in the risk of exacerbations after stopping versus continuing mepolizumab. The subgroup of patients with two or more exacerbations in the previous year was identified as potentially observing less protection from clinically significant exacerbations with mepolizumab, with no treatment benefit observed in this patient subgroup. However, it should be stressed that this subgroup of patients was small and identified following post hoc subgroup analysis, so this finding should be interpreted with caution and considered as hypothesis generating. Further studies are required to understand the impact of discontinuing mepolizumab in patients with two or more exacerbations in the previous year while receiving continuous treatment.

No new safety concerns were observed in this study population compared with those seen in previous studies [1, 3-8]. Additionally, rates of adverse events were similar for patients who stopped mepolizumab and then switched back to mepolizumab treatment following an exacerbation, compared with those who received mepolizumab continuously throughout the study.

One strength of the current study is the monitoring and maintenance of background therapy throughout part C, with no changes recommended per protocol. However, patients were selected from a population who had completed previous mepolizumab clinical trials; therefore, patient recruitment was biased toward those who responded to mepolizumab, and those without adverse events leading to discontinuation from the previous studies. In addition, only $~ 50 \%$ of patients from COLUMBA and COSMEX were enrolled in 
COMET. Patients may have been reluctant to enrol on a placebo-controlled study, particularly in those countries where mepolizumab became commercially available prior to the start of COMET. In addition, patients may also have experienced an amelioration of disease activity after long-term mepolizumab treatment, which may have led to a reduction or discontinuation of their background medications; as patients had to be adherent on asthma controller medication/therapy throughout the previous open-label mepolizumab studies to be eligible for COMET, this may have reduced enrolment. A further limitation was the very small proportion of black patients included in COMET owing to the limited representation of this population in the parent phase III trials. Additionally, while worsening of ACQ-5 and SGRQ scores and worsening $\mathrm{FEV}_{1}$ were seen at week 12 for patients who stopped mepolizumab, this study was not designed to be powered to measure statistical significance for these end-points.

Furthermore, results are based on lower numbers of patients at the later versus earlier time points within part $\mathrm{C}$ with a subset of patients from both arms having discontinued double-blind treatment and switched to part D. This is probably due to the study design, which called for an investigator assessment for patients who experienced a clinically significant exacerbation during part $\mathrm{C}$ to determine whether a switch to open-label mepolizumab in part $\mathrm{D}$ would be appropriate. This issue was mitigated by the use of a hypothetical estimand strategy applied in the handling of the intercurrent event of discontinuation of double-blind treatment. Therefore, the treatment effects reported in part C refer to the outcomes if all patients had continued to take double-blind treatment throughout the 52-week double-blind period. Analysing an annualised exacerbation rate during the double-blind treatment period (part C) was not possible, due to patients being permitted to switch to open-label treatment following their first exacerbation, which meant that repeat exacerbations were not expected by study design.

The investigator assessments during part $\mathrm{C}$ may also have contributed to the low number of exacerbations requiring hospitalisation and/or ED visit, as patients may have been switched to open-label mepolizumab before a more severe exacerbation occurred. Due to the rarity of these more severe exacerbation events during part $\mathrm{C}$, which was consistent with prior trials of mepolizumab [4, 6, 8], any differences between treatment groups should be interpreted with caution. Finally, as this is a double-blind randomised controlled trial, the decisions taken to stop mepolizumab or switch to open-label treatment may not reflect routine clinical practice where the patient's treatment is known, as both patients and physicians were blinded to whether they were on mepolizumab or placebo. Consequently, the finding that of the 45 patients receiving mepolizumab in part $\mathrm{C}$ who switched to part D, 42 switched due to a reported lack of efficacy (in the opinion of the investigator) should be interpreted with caution. Of these 42 patients, 41 also reported exacerbation as a subreason for switching. It is possible that investigators may have switched patients to open-label treatment at the first opportunity (i.e. following their first clinically significant exacerbation) as permitted by study design; this would have enabled patients to return to their established effective therapy, which has been received for at least the prior 3 years. Indeed, most patients who switched to part D to continue mepolizumab in an open-label setting continued mepolizumab treatment until study end, with few withdrawals.

In conclusion, by 12 weeks (16 weeks after the last dose of open-label mepolizumab) patients who stopped long-term ( $\geqslant 3$ years) mepolizumab treatment had a shorter time to first clinically significant exacerbation, an increase in blood eosinophil counts back to pre-treatment levels, and a reduction in asthma control, quality of life and lung function compared with those who continued mepolizumab. These results support continued mepolizumab treatment having sustained clinical benefits in patients with severe eosinophilic asthma and further support that blood eosinophils are a suitable biomarker for treatment response to mepolizumab.

Acknowledgements: Medical writing and editorial support (in the form of writing assistance, including development of the initial draft based on author direction, assembling tables and figures, collating authors' comments, grammatical editing, and referencing) were provided by Elizabeth Hutchinson of Fishawack Indicia Ltd, UK, part of Fishawack Health (funded by GlaxoSmithKline).

This study is registered at Clinicaltrials.gov with identifier NCT02555371. Anonymised individual participant data and study documents can be requested for further research from www.clinicalstudydatarequest.com.

Author contributions: M.J. Gilson and R.G. Price were involved in the conception or design of the work; W.C. Moore, O. Kornmann, M. Humbert, C. Poirier, E.H. Bel, N. Kaneko and M.C. Liu were involved in the acquisition of data; all authors were involved in drafting the work or revising it critically for important intellectual content (i.e. data analysis and interpretation); and all authors agreed to the submission and to be accountable for all aspects of the work. 
Conflict of interest: W.C. Moore reports that the study and writing support was funded by GlaxoSmithKline; and has received funding for clinical research and personal fees for participation in advisory boards from GSK, AstraZeneca and Sanofi Regeneron, outside the submitted work. O. Kornmann reports that the study and writing support was funded by GlaxoSmithKline; and has received personal fees from AstraZeneca, GSK, Novartis, Boehringer Ingelheim, Sanofi Aventis, and Roche, outside the submitted work. M. Humbert reports that the study and writing support was funded by GlaxoSmithKline; and has received personal fees for consultancy services and speaking at conferences, and participation in clinical research projects with AstraZeneca, GSK, Novartis, Roche, Sanofi Regeneron and TEVA; and has a research grant from GSK, outside the submitted work. C. Poirier reports that the study and writing support was funded by GlaxoSmithKline; and has received personal fees from GSK, Novartis, Sanofi, and Boehringer Ingelheim, outside the submitted work. E.H. Bel reports that the study and writing support was funded by GlaxoSmithKline; and grants from GSK and Teva, personal fees from AstraZeneca, GSK, Novartis, Sanofi/Regeneron, Sterna Biologicals and Chiesi, outside the submitted work. N. Kaneko reports that the study and writing support was funded by GlaxoSmithKline. S.G. Smith reports that the study and writing support was funded by GlaxoSmithKline; and is an employee of GSK and owns stocks/shares. N. Martin reports that the study and writing support was funded by GlaxoSmithKline; and is an employee of GSK and owns stocks/ shares. M.J. Gilson reports that the study and writing support was funded by GlaxoSmithKline; and is an employee of GSK and owns stocks/shares. R.G. Price reports that the study and writing support was funded by GlaxoSmithKline; and is an employee of GSK and owns stocks/shares. E.S. Bradford reports that the study and writing support was funded by GlaxoSmithKline; and was an employee at GSK at the time of the study. M.C. Liu reports that the study and writing support was funded by GlaxoSmithKline; and has received grants for clinical trials from Boehringer Ingelheim, GSK, MedImmune, Mereo BioPharm, and Gossamer Bio, outside the submitted work.

Support statement: This study was funded by GlaxoSmithKline (GSK ID: 201810/NCT02555371). The study sponsor had a role in study design, data collection, analysis and interpretation, and in the writing of the report. The sponsor did not place any restrictions on access to data or statements made in the report. Authors had full access to all study data and had final responsibility to submit for publication. Funding information for this article has been deposited with the Crossref Funder Registry.

\section{References}

1 Pavord ID, Korn S, Howarth $\mathrm{P}$, et al. Mepolizumab for severe eosinophilic asthma (DREAM): a multicentre, double-blind, placebo-controlled trial. Lancet 2012; 380: 651-659.

2 Haldar P, Brightling CE, Hargadon B, et al. Mepolizumab and exacerbations of refractory eosinophilic asthma. N Engl J Med 2009; 360: 973-984.

3 Bel EH, Wenzel SE, Thompson PJ, et al. Oral glucocorticoid-sparing effect of mepolizumab in eosinophilic asthma. N Engl J Med 2014; 371: 1189-1197.

4 Ortega HG, Liu MC, Pavord ID, et al. Mepolizumab treatment in patients with severe eosinophilic asthma. N Engl J Med 2014; 371: 1198-1207.

5 Chupp GL, Bradford ES, Albers FC, et al. Efficacy of mepolizumab add-on therapy on health-related quality of life and markers of asthma control in severe eosinophilic asthma (MUSCA): a randomised, double-blind, placebo-controlled, parallel-group, multicentre, phase 3b trial. Lancet Respir Med 2017; 5: 390-400.

6 Lugogo N, Domingo C, Chanez P, et al. Long-term efficacy and safety of mepolizumab in patients with severe eosinophilic asthma: a multi-center, open-label, phase IIIb study. Clin Ther 2016; 38: 2058-2070.

7 Khatri S, Moore W, Gibson PG, et al. Assessment of the long-term safety of mepolizumab and durability of clinical response in patients with severe eosinophilic asthma. J Allergy Clin Immunol 2019; 143: 1742-1751.

8 Khurana S, Brusselle GG, Bel EH, et al. Long-term safety and clinical benefit of mepolizumab in patients with the most severe eosinophilic asthma: the COSMEX study. Clin Ther 2019; 41: 2041-2056.

$9 \quad$ United States Food and Drug Admininstration. Mepolizumab (NUCALA) Prescribing Information. 2019. www. gsksource.com/pharma/content/dam/GlaxoSmithKline/US/en/Prescribing_Information/Nucala/pdf/NUCALA-PIPIL.PDF Date last accessed: 15 January 2020.

10 European Medicines Agency. Mepolizumab (NUCALA) Summary of Product Characteristics. 2019. www.ema. europa.eu/en/documents/product-information/nucala-epar-product-information_en.pdf Date last accessed: 15 January 2020.

11 Flood-Page P, Menzies-Gow A, Phipps S, et al. Anti-IL-5 treatment reduces deposition of ECM proteins in the bronchial subepithelial basement membrane of mild atopic asthmatics. J Clin Invest 2003; 112: 1029-1036.

12 Menzies-Gow A, Flood-Page P, Sehmi R, et al. Anti-IL-5 (mepolizumab) therapy induces bone marrow eosinophil maturational arrest and decreases eosinophil progenitors in the bronchial mucosa of atopic asthmatics. J Allergy Clin Immunol 2003; 111: 714-719.

13 Haldar P, Brightling CE, Singapuri A, et al. Outcomes after cessation of mepolizumab therapy in severe eosinophilic asthma: a 12-month follow-up analysis. J Allergy Clin Immunol 2014; 133: 921-923. 
14 Caminati M, Polk B, Rosenwasser LJ. What have recent advances in therapy taught us about severe asthma disease mechanisms? Expert Rev Clin Immunol 2019; 15: 1145-1153.

15 Juniper EF, Svensson K, Mörk AC, et al. Measurement properties and interpretation of three shortened versions of the asthma control questionnaire. Respir Med 2005; 99: 553-558.

16 Jones PW. Interpreting thresholds for a clinically significant change in health status in asthma and COPD. Eur Respir J 2002; 19: 398-404.

17 Ortega HG, Yancey SW, Mayer B, et al. Severe eosinophilic asthma treated with mepolizumab stratified by baseline eosinophil thresholds: a secondary analysis of the DREAM and MENSA studies. Lancet Respir Med 2016; 4: 549-556.

18 Yancey SW, Keene ON, Albers FC, et al. Biomarkers for severe eosinophilic asthma. J Allergy Clin Immunol 2017; 140: 1509-1518.

19 Ortega H, Menzies-Gow A, Llanos JP, et al. Rapid and consistent improvements in morning PEF in patients with severe eosinophilic asthma treated with mepolizumab. Adv Ther 2018; 35: 1059-1068.

20 Ortega $\mathrm{H}$, Lemiere $\mathrm{C}$, Llanos JP, et al. Outcomes following mepolizumab treatment discontinuation: real-world experience from an open-label trial. Allergy Asthma Clin Immunol 2019; 15: 37.

21 Wenzel S, Ford L, Pearlman D, et al. Dupilumab in persistent asthma with elevated eosinophil levels. $N$ Engl J Med 2013; 368: 2455-2466.

22 Peters MC, Mauger D, Ross KR, et al. Evidence for exacerbation-prone asthma and predictive biomarkers of exacerbation frequency. Am J Respir Crit Care Med 2020; 202: 973-982.

23 Kuruvilla ME, Lee FE, Lee GB. Understanding asthma phenotypes, endotypes, and mechanisms of disease. Clin Rev Allergy Immunol 2019; 56: 219-233.

24 Fleming L, Tsartsali L, Wilson N, et al. Sputum inflammatory phenotypes are not stable in children with asthma. Thorax 2012; 67: 675-681.

25 Green RH, Pavord I. Stability of inflammatory phenotypes in asthma. Thorax 2012; 67: 665-667.

26 Silkoff PE, Laviolette M, Singh D, et al. Longitudinal stability of asthma characteristics and biomarkers from the Airways Disease Endotyping for Personalized Therapeutics (ADEPT) study. Respir Res 2016; 17: 43.

27 Schatz M, Meckley LM, Kim M, et al. Asthma exacerbation rates in adults are unchanged over a 5-year period despite high-intensity therapy. J Allergy Clin Immunol Pract 2014; 2: 570-574.

28 Ledford D, Busse W, Trzaskoma B, et al. A randomized multicenter study evaluating Xolair persistence of response after long-term therapy. J Allergy Clin Immunol 2017; 140: 162-169. 